\title{
High-pressure structural phase transitions in semiconducting niobium dioxide
}

\author{
J. Haines, J. M. Léger, and A. S. Pereira* \\ Centre National de la Recherche Scientifique, Laboratoire de Physico-Chimie des Matériaux, 1, Place Aristide Briand, \\ 92190 Meudon, France \\ D. Häusermann and M. Hanfland \\ European Synchrotron Radiation Facility, Boîte Postale 220, 38043 Grenoble, France
}

(Received 2 November 1998)

\begin{abstract}
Niobium dioxide was studied up to $47 \mathrm{GPa}$ by angle-dispersive, $\mathrm{x}$-ray powder diffraction. Semiconducting $\alpha-\mathrm{NbO}_{2}$ (space group $I 4_{1} / a, Z=32$ ) was found to transform to $\beta-\mathrm{NbO}_{2}$ (space group $I 4_{1}, Z=16$ ) above 5 $\mathrm{GPa}$. Both phases have distorted rutile-type structures and the transition involves a change in the long-range ordering of the metal-metal bonds. A further transition was observed above $8 \mathrm{GPa}$ to a baddeleyite-related structure with $a=9.975(1), b=9.963(1), c=9.933(1) \AA, \beta=104.023(4)^{\circ}$, and $Z=32$ at $12.8 \mathrm{GPa}$. The doubling of the unit cell along $\mathbf{a}, \mathbf{b}$, and $\mathbf{c}$ and the larger monoclinic angle with respect to a baddeleyite-type cell are due to the presence of metal-metal bonds. The $d^{1}$ cation dioxide, $\mathrm{NbO}_{2}$, is thus shown to behave in a similar way to $d^{0}$ cation dioxides, which follow a phase transition sequence involving the baddeleyite-type structure. [S0163-1829(99)13021-2]
\end{abstract}

\section{INTRODUCTION}

Semiconducting $\alpha-\mathrm{NbO}_{2}$ has a distorted rutile-type structure (space group $I 4_{1} / a, \quad Z=32, \quad a=13.7020 \AA, \quad c$ $=5.9850 \AA$ ) under ambient conditions. ${ }^{1-4}$ The structural distortion is due to the presence of $\mathrm{Nb}-\mathrm{Nb}$ bond pairs, which lie across the shared edges linking the $\mathrm{NbO}_{6}$ octahedra along the c direction. The niobium ions are displaced from the center of the distorted oxygen octahedra yielding polyhedral $\mathrm{Nb}-\mathrm{O}$ distances ranging from 1.91 to $2.25 \AA$ and there are the two distinct $\mathrm{Nb}-\mathrm{Nb}$ distances of 2.71 and $3.30 \AA^{4} \quad \mathrm{~A}$ semiconductor-metal transition ${ }^{5-9}$ occurs at $1081 \mathrm{~K}$ to a rutile-type phase (space group $P 4_{2} / \mathrm{mnm}, Z=2, \quad a$ $=4.8463 \AA, c=3.0315 \AA$ at $1300 \mathrm{~K}) .{ }^{4}$ The $c / a$ ratio is low for a rutile-type structure and the $\mathbf{c}$ repeat corresponds to a $\mathrm{Nb}-\mathrm{Nb}$ bond. The conductivity in this phase remains semiconductorlike in the a direction, whereas it is metallic-like along $\mathbf{c}^{9}{ }^{9}$ The octahedra in rutile-type $\mathrm{NbO}_{2}$ are less distorted than in $\alpha-\mathrm{NbO}_{2}$, with two Nb-O distances of $2.005 \AA$ and four of $2.079 \AA$. The lattice parameters of the lowtemperature phase $(\alpha)$ can be related to those of the hightemperature rutile-type phase $(r)$ as follows: $\mathbf{a}_{1 \alpha}=2\left(\mathbf{a}_{1 r}\right.$ $\left.-\mathbf{a}_{2 r}\right), \mathbf{a}_{2 \alpha}=2\left(\mathbf{a}_{1 r}+\mathbf{a}_{2 r}\right), \mathbf{c}_{\alpha}=2 \mathbf{c}_{r}$. The transition is second order and is driven by a soft mode with wave vector $\mathbf{q}$ $=(1 / 4,1 / 4,1 / 2){ }^{8}$

A second-form of $\mathrm{NbO}_{2}, \beta-\mathrm{NbO}_{2}$, has been prepared by chemical transport reactions. ${ }^{10} \beta-\mathrm{NbO}_{2}$ also has a distorted rutile-type structure (space group $I 4_{1}, Z=16, a=9.693 \AA$, $c=5.985 \AA$ ) and differs from $\alpha-\mathrm{NbO}_{2}$ in the long-range ordering of the $\mathrm{Nb}-\mathrm{Nb}$ pairs. The $\mathrm{Nb}-\mathrm{Nb}$ distances are essentially identical to those in $\alpha-\mathrm{NbO}_{2}$ and the polyhedral $\mathrm{Nb}-\mathrm{O}$ distances range from 1.904 to $2.211 \AA$. The lattice parameters of this phase $(\beta)$ are related to the high-temperature, rutile-type phase as follows: $\mathbf{a}_{\beta}=2 \mathbf{a}_{r}, \mathbf{c}_{\beta}=2 \mathbf{c}_{r}$. The relative volumes of $\alpha-\mathrm{NbO}_{2}$ and $\beta-\mathrm{NbO}_{2}$ are essentially identical as are the average $\mathrm{Nb}-\mathrm{O}$ polyhedral distances. Madelung potential lattice energy calculations indicate a very small difference between $\alpha-\mathrm{NbO}_{2}(12858 \mathrm{~kJ} / \mathrm{mol})$ and $\beta-\mathrm{NbO}_{2}$ $(12866 \mathrm{~kJ} / \mathrm{mol})$. This indicates that $\alpha-\mathrm{NbO}_{2}$ is the stable phase under ambient conditions.

Ultrasonic $^{11}$ and resistivity ${ }^{12,13}$ measurements have been performed on $\alpha-\mathrm{NbO}_{2}$ at high pressure. Ultrasonic measurements up to $0.5 \mathrm{GPa}$ indicate a softening of the $C_{\mathrm{QS}}$ and $C_{66}$ elastic constants and the results suggest the possibility of a transition at higher pressure to a monoclinic phase. ${ }^{11}$ Highpressure resistivity measurements indicate a phase transition; however, there is a large difference between the reported transition pressures of either $2.7 \mathrm{GPa}$ (Ref. 12) or $8.5 \mathrm{GPa} .{ }^{13}$ In the second of these studies, ${ }^{13}$ the phase above $8.5 \mathrm{GPa}$ was found to be a narrow gap semiconductor and it was concluded that a weak metal-metal bond is retained in this phase. The semiconductor-metal transition temperature was found to decrease from $1067 \mathrm{~K}$ (at $0.1 \mathrm{MPa}$ ) by $16 \mathrm{~K} / \mathrm{GPa}$ with pressure. ${ }^{13}$ This and the metallic nature of the rutile phase indicate that it cannot be considered as a candidate for the ambient-temperature, high-pressure phase found above $8.5 \mathrm{GPa}$.

Investigations of rutile-structured compounds ${ }^{14-19}$ at high pressure indicate that these compounds undergo transitions along phase-transformation sequences passing via either the monoclinic baddeleyite structure or the cubic $\mathrm{Pa} \overline{3}$ structure depending on the electronic configuration of the metal cation. Baddeleyite and baddeleyite-related structures, and thus the pathway passing via this structure, are characteristic of $d^{0}$ cation compounds. ${ }^{14,18,19}$ Other compounds adopt the $\mathrm{Pa} \overline{3}$ structure at high pressure. The high-pressure behavior of distorted-rutile-type $\alpha-\mathrm{NbO}_{2}$, in which the cation $\mathrm{Nb}$ has a $4 d^{1}$ electronic configuration, is of considerable interest in relation to the effect of metal-metal bonds on these phase transition sequences and the behavior of $d^{1}$ cation compounds.

\section{EXPERIMENTAL PROCEDURE}

Powdered $\alpha-\mathrm{NbO}_{2}$ (Alfa Products, purity 99+) was used for all high-pressure experiments. A stainless steel gasket, preindented to a thickness of $120 \mu \mathrm{m}$ with a $200 \mu \mathrm{m}$ diameter hole was used for laboratory experiments. The stainless 
steel gasket used in the synchrotron experiments was preindented to a thickness of $38 \mu \mathrm{m}$ and had a hole diameter of $100 \mu \mathrm{m}$. Silicone grease was used as a pressure transmitting medium for laboratory experiments and a mixture of 16:3:1 methanol:ethanol:water was used for synchrotron experiments. Samples were loaded along with ruby powder and the pressure-transmitting medium in the gasket placed between the anvils of a diamond anvil cell (DAC). Pressures were measured based on the shifts of the ruby $R_{1}$ and $R_{2}$ fluorescence lines. ${ }^{20}$

Laboratory x-ray diffraction patterns were obtained on an imaging plate placed at $96.36 \mathrm{~mm}$ from the sample using zirconium-filtered molybdenum radiation from a microfocus tube collimated to $130 \mu \mathrm{m} \times 130 \mu \mathrm{m}$. Exposure times were typically of between $24-60 \mathrm{~h}$. A dac in which the rear diamond was mounted over a $16^{\circ}$ wide slit allowing access to an angular range $4 \theta=80^{\circ}$ was used for these experiments. Heating using a $50 \mathrm{~W}$ Nd:YAG laser was performed at the highest pressure attained, $47 \mathrm{GPa}$, and at $16.9 \mathrm{GPa}$ on decompression to reduce anisotropic stress and, in addition, at $47 \mathrm{GPa}$ to attempt to induce a further phase transition. Additional exposures were obtained using the same installation on the starting material in a capillary tube and on the material recovered after the experiment in the gasket with sample to plate distances of 122.34 and $92.41 \mathrm{~mm}$, respectively.

Synchrotron x-ray diffraction experiments were performed on beamline ID30 at the ESRF using a dac with a full conical aperture $\left(4 \theta=56^{\circ}\right)$. X-ray wavelengths of 0.4241 and $0.4226 \AA$ were selected using a $\mathrm{Si}(111)$ Laue-Bragg monochromator. The incident beam diameter was $20 \mu \mathrm{m}$ at half maximum. Image plates were placed at a distance of $398.74 \mathrm{~mm}$ from the sample and exposure times were of 10 to $15 \mathrm{~min}$.

The observed intensities on the imaging plates were integrated as a function of $2 \theta$ in order to give conventional onedimensional diffraction profiles. All diffraction data were analyzed by full profile fitting to obtain unit cell constants using the program FULLPROF. ${ }^{21}$ Full experimental details and a comparison between synchrotron and conventional source, high-pressure, x-ray powder diffraction methods are given elsewhere. ${ }^{22}$ All figures in parentheses refer to estimated standard deviations (ESD).

\section{RESULTS}

\section{A. $\alpha-\mathrm{NbO}_{2}$ to $\beta-\mathrm{NbO}_{2}$ transition}

The following cell constants were refined for the sample of $\alpha-\mathrm{NbO}_{2}$ under ambient conditions: $a=13.7000(5) \AA$ and $c=5.9878(4) \AA$. These values are in good agreement with those given in Ref. 4. No reflections other than those due to $\alpha-\mathrm{NbO}_{2}$ were observed and the relative intensities were in agreement with those calculated using the atomic coordinates obtained from single crystal neutron diffraction experiments. ${ }^{2}$ It should be noted that in $\alpha-\mathrm{NbO}_{2}$, there are two crystallographically independent niobium ions and four crystallographically-independent oxygen ions, all of which lie on the general positions, $16 f$. Due to the resulting large number of free positional parameters, particularly for the oxygen ions, structure refinements could not be performed.

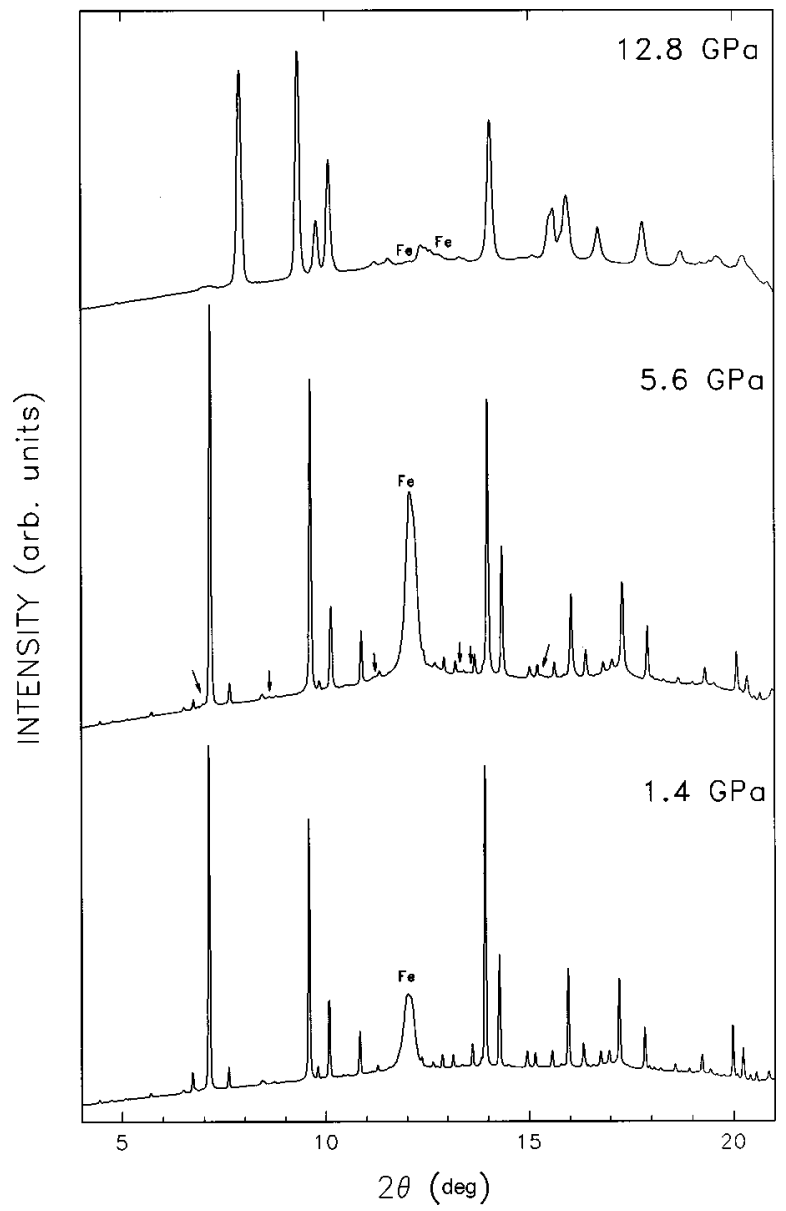

FIG. 1. X-ray diffraction patterns of $\mathrm{NbO}_{2}$ at high pressure $(\lambda$ $=0.4241 \AA): \quad \alpha-\mathrm{NbO}_{2}$ at $1.4 \mathrm{GPa}$, a mixture of $\alpha-\mathrm{NbO}_{2}$ and $\beta-\mathrm{NbO}_{2}$ at $5.6 \mathrm{GPa}$ (arrows indicate the most intense nonrutile-type subcell reflections of $\beta-\mathrm{NbO}_{2}: 211,301,411,431,213$, and 323 with increasing $2 \theta$ ) and monoclinic $\mathrm{NbO}_{2}$ at $12.8 \mathrm{GPa}$. Fe indicates reflections arising from the stainless steel gasket.

Above $5 \mathrm{GPa}$, the weak reflections, which do not belong to the rutile-type subcell of $\alpha-\mathrm{NbO}_{2}$, decreased in intensity, while the subcell reflections remained intense. New diffraction lines were detected and were found to increase in intensity upon further compression; see Fig. 1. These new lines could readily be indexed as the $211,301,411,431,213$, and 323 reflections of $\beta-\mathrm{NbO}_{2}$. These are the most intense reflections of this phase that are not due to the rutile-type subcell. No asymmetry was observed in the line shape of the common subcell reflections, thus no volume change between the two phases was apparent and the following cell constants $a=13.5873(2) \AA$ and $c=5.9482(1) \AA$ were obtained for $\alpha-\mathrm{NbO}_{2}$ and $a=9.6076(1) \AA$ and $c=5.9482(1) \AA$ for $\beta-\mathrm{NbO}_{2}$ at $5.6 \mathrm{GPa}$; see Fig. 2. Both group theory ${ }^{10}$ and the pressure range of intergrowth between the two phases indicate that this transition is first order, and thus there must be a volume change. No volume change was detected in the present experiment, consequently, it is less than $0.2 \%$.

\section{B. Transition to the high-pressure monoclinic phase}

A major change to the diffraction pattern was observed at $8.3 \mathrm{GPa}$. All the $\beta-\mathrm{NbO}_{2}$ reflections decreased in intensity 

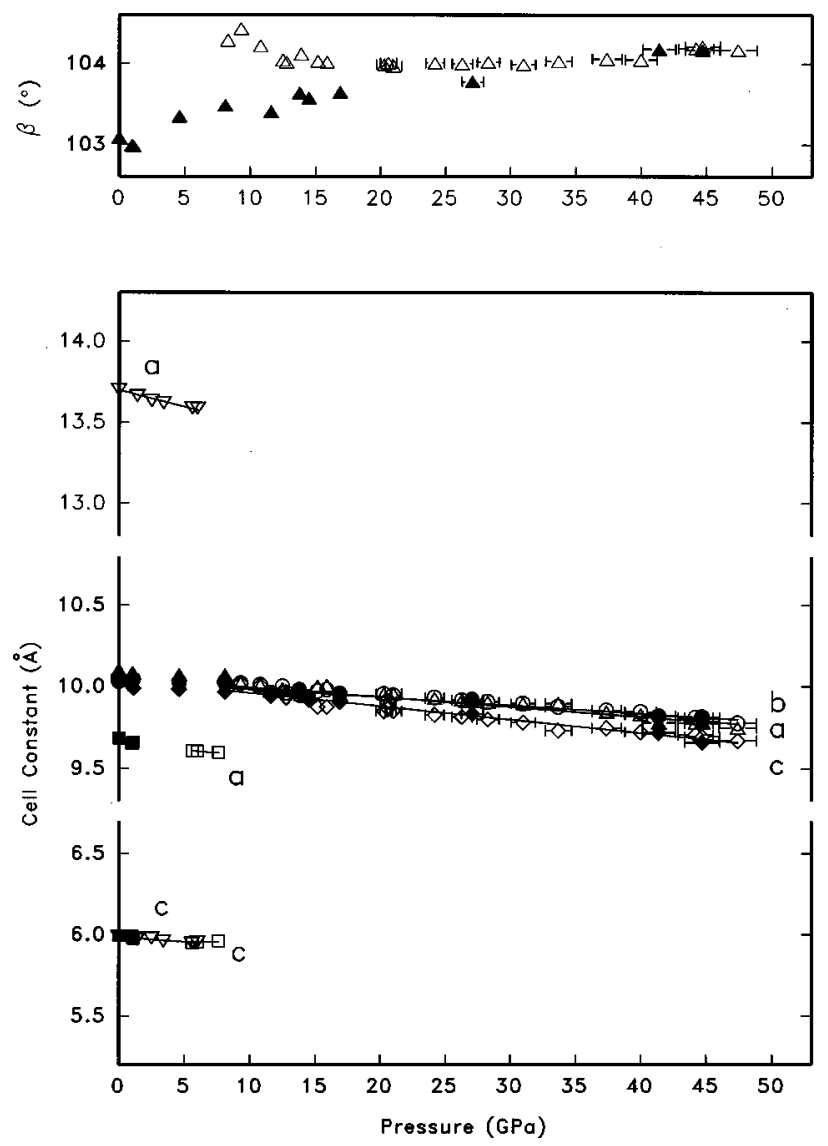

FIG. 2. Cell constants of $\mathrm{NbO}_{2}$ as a function of pressure. Open symbols refer to points obtained upon compression and solid symbols to those obtained on decompression. Symbols $\nabla$ and $\square$ correspond to $\alpha-\mathrm{NbO}_{2}$ and $\beta-\mathrm{NbO}_{2}$ and $\triangle(a, \beta), \bigcirc(b)$, and $\diamond(c)$ to monoclinic $\mathrm{NbO}_{2}$, respectively. Error bars are shown where the error is larger than the symbol size. Solid lines represent least squares fits to the data.

and a large number of new diffraction lines appeared. The transition was found to be essentially complete by $12.8 \mathrm{GPa}$ with only about $1 \% \beta-\mathrm{NbO}_{2}$ remaining. The intense diffraction lines in the diffraction pattern of this new phase could readily be indexed by analogy to the high-pressure, baddeleyite-type structure $\left(P 2_{1} / c, Z=4\right)$ of $\mathrm{TiO}_{2} .{ }^{14}$ The relative intensities of these intense lines are very similar to those calculated for baddeleyite-type $\mathrm{TiO}_{2}$. The cell constants of monoclinic $\mathrm{TiO}_{2}$ at $20.3 \mathrm{GPa}$ are $a=4.64 \AA, b$ $=4.76 \AA, c=4.81 \AA$, and $\beta=99.2^{\circ}$. Profile fitting yielded the following constants for the monoclinic cell of $\mathrm{NbO}_{2}$ at $12.8 \mathrm{GPa}: a=4.9877(5) \AA, \quad b=4.9814(3), c$ $=4.9663(4)$, and $\beta=104.02(4)^{\circ}$. The monoclinic angle is larger than in baddeleyite-type $\mathrm{TiO}_{2}$ and the difference between the cell constants $a, b$, and $c$ is greatly reduced. A baddeleyite-related structure with a $\beta$ angle of $103.581^{\circ}$ has recently been reported ${ }^{23}$ for the high-pressure, hydrogenbonded phase of $\mathrm{Ca}(\mathrm{OD})_{2}$. In the present case, the large monoclinic angle is due to the presence of $\mathrm{Nb}-\mathrm{Nb}$ bonds. Attempts were made to refine the structure of the highpressure phase of $\mathrm{NbO}_{2}$ starting from a baddeleyite-type structural model. Good agreement was obtained for the strong reflections and the overall Bragg $R$ factor was $4 \%$; however, several very weak peaks observed experimentally were absent from the calculated pattern (see following paragraph). The final niobium ion positions were displaced by 0.01 along $x, y$, and $z$ from distorted fcc (face centered cubic) -like positions and fixing the metal positions to $(0.25,0$, $0.25)$ had almost no effect on the profile. It can be noted that in baddeleyite $\left(\mathrm{ZrO}_{2}\right)$ the metal ions at $(0.2758,0.0411$, 0.2082 ) are displaced by up to 0.041 from distorted fcc-type positions. ${ }^{24}$ The cation displacements are significantly smaller in monoclinic $\mathrm{NbO}_{2}$ as is the difference between the cell constants and the cations lie essentially on a monoclinically-sheared fcc sublattice. Assuming these latter positions $(0.25,0,0.25), \mathrm{Nb}-\mathrm{Nb}$ distances of $3.06,3.52$, and $3.92 \AA$ are obtained at $12.8 \mathrm{GPa}$. The shortest $\mathrm{Nb}-\mathrm{Nb}$ distances lie along the [101] direction and yield chains of bonded metal ions. In a cubic cell with the same volume, all $\mathrm{Nb}-\mathrm{Nb}$ distances would be of $3.48 \AA$, whereas in a baddeleyite-type cell with a $\beta$ angle of $99^{\circ}$, the minimum $\mathrm{Nb}-\mathrm{Nb}$ distance would be $3.21 \AA$. It can be seen that the formation of metal-bonded chains increases the monoclinic angle $\beta$.

Upon close inspection of the high-pressure diffraction patterns of monoclinic $\mathrm{NbO}_{2}$, a series of weak peaks with relative intensities of less than $4 \%$ were identified; see Table I. In order to index these weak peaks, it was necessary to double the unit cell along $a, b$, and $c$ yielding the following cell constants: $a=9.975(1), \quad b=9.963(1), c=9.933(1)$ $\AA, \beta=104.023(4)^{\circ}$ at $12.8 \mathrm{GPa}$. The observed reflections in Table I respect the following reflection conditions: $0 k 0$ : $k=2 n ; h 0 l: h+l=2 n$, indicating that if the reflections not observed are truly absent, the space group would be $P 2_{1} / n$. In a $P 2_{1} / n$ cell with $Z=32$, there would be 8 crystallographically-independent niobium ions and 16 crystallographically-independent oxygen ions all on general $4 e$ positions. Such a structure is too complex to be determined using high-pressure, $\mathrm{x}$-ray diffraction data. Due to the low intensity of the superlattice peaks, the displacement of the niobium ions from distorted-fcc lattice sites would be small, probably of the order of up to 0.01 . The resulting effect on short $\mathrm{Nb}-\mathrm{Nb}$ distances $(3.06 \AA)$ would be a modulation of approximately $\pm 1-2 \%$. At least one of these $\mathrm{Nb}-\mathrm{Nb}$ distances would be longer than the distances of $3.0315 \AA$ in the metallic high-temperature phase, but the difference between the two distances would be significantly less than for the two alternating distances of 2.71 and $3.30 \AA$ in semiconducting $\alpha-\mathrm{NbO}_{2}$ and $\beta-\mathrm{NbO}_{2}$. The present results are thus consistent with the large decrease in resistivity at the transition to monoclinic $\mathrm{NbO}_{2}$ and the observation that this phase is a narrow gap semiconductor. ${ }^{13}$ If this phase were metallic, the $\mathrm{Nb}-\mathrm{Nb}$ distances in the chains would be equal and there would be no superstructure.

The oxide anions almost certainly occupy a baddeleyitelike arrangement, which is distorted due to the presence of metal-metal bonds. Neutron diffraction data from this phase are required in order to determine the exact oxygen positions.

In the experiment using the methanol:ethanol:water mixture as a hydrostatic pressure transmitting medium, the relative intensities of the diffraction lines from the monoclinic phase remained effectively constant over the pressure range 
TABLE I. High-pressure diffraction data obtained from fullprofile fitting for monolinic $\mathrm{NbO}_{2}$ at $12.8 \mathrm{GPa}: a=9.975(1), b$ $=9.963(1), c=9.933(1) \AA, \beta=104.023(4)^{\circ}$, and $Z=32$. (Note: only the reflections in bold type are present for a baddeleyitetype subcell.)

\begin{tabular}{lrrrrr}
\hline \hline$h k l$ & $\mathrm{~d}(\AA)$ & $I_{\text {obs }}$ & $h k l$ & $d(\AA)$ & $I_{\text {obs }}$ \\
\hline 020 & 4.981 & 0.5 & $45 \overline{1}$ & 1.557 & 3.6 \\
$\mathbf{2 2 0}$ & 3.471 & 0.5 & $54 \overline{1}$ & 1.556 & 2.6 \\
$\mathbf{0 2 2}$ & 3.463 & 0.7 & $\mathbf{2 4 4}$ & 1.547 & 0.4 \\
$\mathbf{2 2 \overline { 2 }}$ & 3.082 & 100.0 & $\mathbf{4 4 \overline { 4 }}$ & 1.541 & 5.1 \\
$\mathbf{2 2 2}$ & 2.609 & 94.1 & $\mathbf{6 2 0}$ & 1.535 & 1.4 \\
$\mathbf{0 4 0}$ & 2.491 & 22.9 & $\mathbf{4 0 4}$ & 1.532 & 13.4 \\
$\mathbf{4 0 0}$ & 2.420 & 21.5 & $\mathbf{2 6 \overline { 2 }}$ & 1.529 & 22.8 \\
$\mathbf{0 0 4}$ & 2.409 & 24.0 & $\mathbf{6 0 \overline { 4 }}$ & 1.522 & 4.6 \\
$14 \overline{1}$ & 2.374 & 1.7 & $\mathbf{2 6 2}$ & 1.460 & 14.3 \\
$\mathbf{4 2 0}$ & 2.176 & 0.5 & $\mathbf{6 2 \overline { 4 }}$ & 1.455 & 3.9 \\
$\mathbf{4 2 \overline { 2 }}$ & 2.169 & 0.5 & $\mathbf{4 2 \overline { 6 }}$ & 1.452 & 1.1 \\
241 & 2.108 & 2.2 & $\mathbf{2 4} \overline{\mathbf{6}}$ & 1.375 & 3.4 \\
$\mathbf{2 4 \overline { 2 }}$ & 2.103 & 1.3 & $\mathbf{6 2 2}$ & 1.374 & 9.3 \\
$\mathbf{2 0 4}$ & 1.974 & 7.5 & $\mathbf{2 2 6}$ & 1.370 & 9.3 \\
$\mathbf{4 0 \overline { 4 }}$ & 1.961 & 1.0 & $\mathbf{4 6 0}$ & 1.369 & 5.7 \\
150 & 1.952 & 2.7 & $\mathbf{0 6 4}$ & 1.367 & 0.5 \\
051 & 1.951 & 2.7 & $\mathbf{4 6 \overline { 2 }}$ & 1.367 & 0.5 \\
$\mathbf{2 4 2}$ & 1.933 & 5.3 & $\mathbf{4 4 4}$ & 1.305 & 9.1 \\
$\mathbf{2 2 4}$ & 1.835 & 0.7 & $\mathbf{6 4 \overline { 4 }}$ & 1.298 & 1.5 \\
$\mathbf{5 2 \overline { 2 }}$ & 1.831 & 0.8 & $\mathbf{4 4 \overline { 6 }}$ & 1.296 & 1.7 \\
$\mathbf{4 2 \overline { 4 }}$ & 1.825 & 0.6 & $34 \overline{5}$ & 1.286 & 0.4 \\
501 & 1.815 & 0.8 & $33 \overline{7}$ & 1.285 & 0.5 \\
$\mathbf{4 4 0}$ & 1.735 & 29.8 & $\mathbf{4 6 2}$ & 1.272 & 1.8 \\
$\mathbf{0 4 4}$ & 1.732 & 33.3 & $\mathbf{2 6 4}$ & 1.271 & 0.5 \\
$\mathbf{2 4} \overline{\mathbf{4}}$ & 1.729 & 11.8 & $46 \overline{4}$ & 1.267 & 0.8 \\
$20 \overline{6}$ & 1.648 & 0.5 & $\mathbf{6 2 \overline { 6 }}$ & 1.265 & 0.9 \\
$42 \overline{3}$ & 1.643 & 0.3 & 065 & 1.258 & 0.7 \\
$61 \overline{1}$ & 1.635 & 0.3 & 534 & 1.256 & 0.5 \\
$\mathbf{6 0 0}$ & 1.613 & 3.0 & $\mathbf{0 8 0}$ & 1.245 & 8.8 \\
$\mathbf{6 2 \overline { 2 }}$ & 1.571 & 15.2 & $\mathbf{2 0 \overline { 8 }}$ & 1.242 & 0.9 \\
$\mathbf{2 2 \overline { 6 }}$ & 1.565 & 20.1 & & & \\
\hline \hline & & & & & \\
\hline
\end{tabular}

below $15 \mathrm{GPa}$, for which this medium remains liquid. Above this pressure and in the experiment using silicone grease as a pressure-transmitting medium above $12 \mathrm{GPa}, I_{111} / I_{11} \overline{1}$ decreased with increasing pressure. In this second experiment, this ratio decreased from $102 \%$ at $10.8 \mathrm{GPa}$ to $17 \%$ at 47 $\mathrm{GPa}$ with most of this decrease occurring below $20 \mathrm{GPa}$. Similar behavior has been reported for baddeleyite-type $\mathrm{TiO}_{2}$ and was attributed to preferred orientation. ${ }^{25}$ The effect of the pressure transmitting medium in the present case confirms that this behavior is a result of preferred orientation, which appears to be a characteristic of baddeleyite-type structures under nonhydrostatic conditions.

The sample was laser heated to approximately $1000^{\circ} \mathrm{C}$ at $47 \mathrm{GPa}$ in an attempt to induce a further phase transition. No significant changes were observed in the diffraction pattern indicating that no further transition occurs and thus the monoclinic phase remained stable up to the highest pressure reached. A decrease in the separation between the ruby $R_{1}$ and $R_{2}$ lines and a narrowing of their linewidths after heating

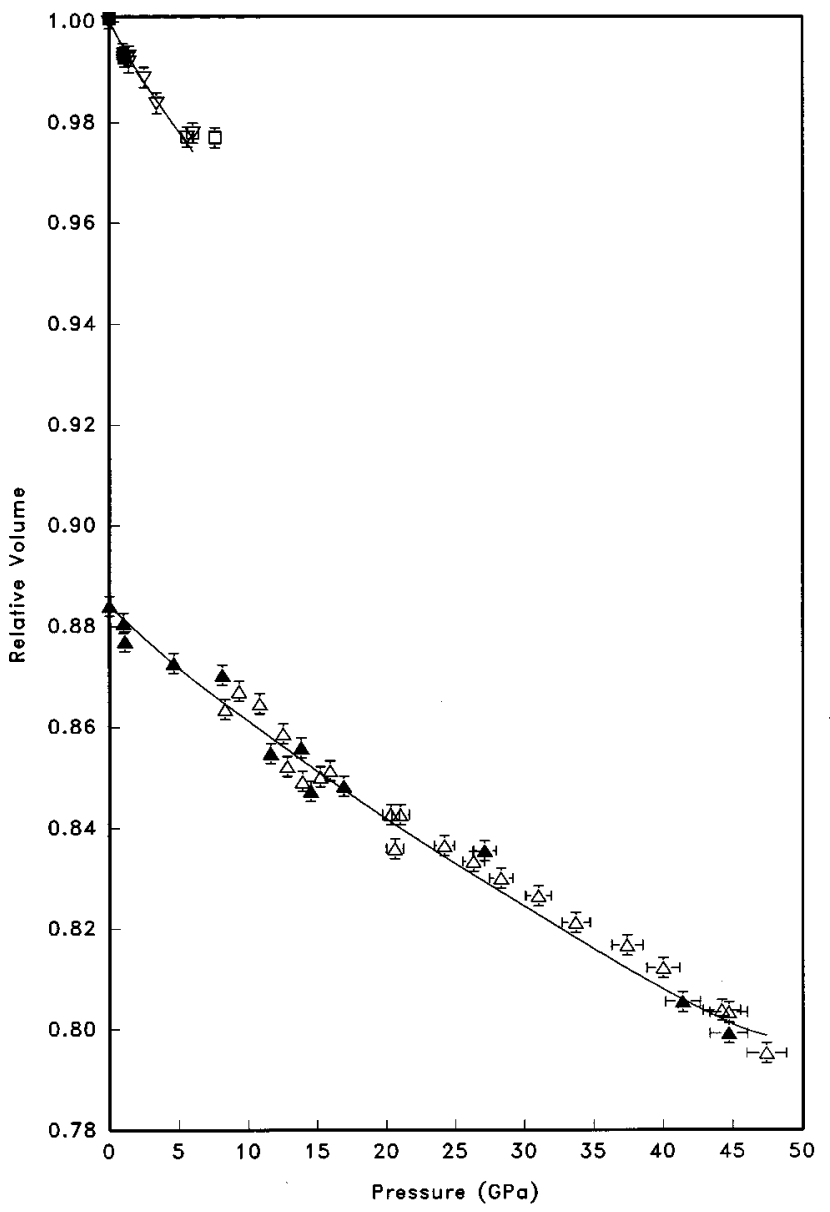

FIG. 3. Relative volume of $\mathrm{NbO}_{2}$ as a function of pressure. Open symbols refer to points obtained upon compression and solid symbols to those obtained on decompression. Symbols $\nabla, \square$, and $\triangle$ correspond to $\alpha-\mathrm{NbO}_{2}, \beta-\mathrm{NbO}_{2}$, and monoclinic $\mathrm{NbO}_{2}$, respectively. Solid lines represent Birch-Murnaghan equations of state using the parameters obtained in this study.

was observed, which indicates that the deviatoric stress experienced by the sample was reduced.

The monoclinic angle $\beta$ increased very slightly with pressure above $12 \mathrm{GPa}$; see Fig. 2. The larger values obtained between 8.3 and $10.8 \mathrm{GPa}$ upon compression are likely to be due to strain resulting from intergrowth with the significant amount of $\beta-\mathrm{NbO}_{2}$ remaining over this pressure range. Upon decompression, a significant and reproducible decrease in the angle $\beta$ was observed irrespective of the pressure transmitting medium used. This effect is much larger than the uncertainty in beta, for which the esd was typically less than $0.02^{\circ}$. It can be noted that the separation between the two strongest reflections, 111 and $11 \overline{1}$, is governed by $\beta$ and thus this parameter is obtained with very high precision by full profile fitting.

Monoclinic $\mathrm{NbO}_{2}$ was found to begin to retransform to $\beta-\mathrm{NbO}_{2}$ at close to $5 \mathrm{GPa}$ (Fig. 3). The material recovered under ambient conditions was $80 \% \beta-\mathrm{NbO}_{2}$ with cell constants, $a=9.686(1) \AA$ and $c=5.994(1) \AA$ and $20 \%$ monoclinic $\mathrm{NbO}_{2}$ with cell constants, $a=10.10(2) \AA, \quad b$ $=10.03(1) \AA, c=10.06(2) \AA$, and $\beta=103.1(1)^{\circ}$. The large ESD's for the monoclinic phase reflect uncertainty due to peak overlap. 


\section{DISCUSSION}

\section{A. Bulk moduli and linear compressibilities and volume changes}

The experimental $P-V$ data (Fig. 3) were fitted using a first-order Birch-Murnaghan equation of state (EOS): ${ }^{26}$

$$
P=1.5 B_{0}\left[\left(V / V_{0}\right)^{-7 / 3}-\left(V / V_{0}\right)^{-5 / 3}\right],
$$

where $B_{0}$ is the bulk modulus at ambient pressure. The firstorder form of the Birch-Murnaghan equation implies that the value of the first pressure derivative of $B_{0}$ is 4 , which is a typical value for a large number of materials. A fit to the data for $\alpha-\mathrm{NbO}_{2}$ yielded a bulk modulus of 230(10) GPa, which is in very good agreement with the value of $236 \mathrm{GPa}$ obtained from ultrasonic measurements ${ }^{27}$ on single-crystal $\mathrm{NbO}_{2}$. The compression of $\alpha-\mathrm{NbO}_{2}$ is anisotropic with $\kappa_{a}$ $=15.3(7) \times 10^{-4} \mathrm{GPa}^{-1}$ and $\kappa_{c}=10.4(10) \times 10^{-4} \mathrm{GPa}^{-1}$. In the case of $\alpha-\mathrm{NbO}_{2}$, the ratio of linear compressibilities along a and $\mathbf{c}$ is 1.47 , whereas it is typically $2-3$ in rutiletype structures. ${ }^{17,18,28,29}$ The relative compression along $\mathbf{c}$ in $\alpha-\mathrm{NbO}_{2}$ is greater than in rutile-type structures due to the structural distortion, which results in the cations being arranged in zig-zag chains with alternating short and long metal-metal contacts along c, instead of straight chains with a single metal-metal distance as in the rutile structure. Compression thus can occur along $\mathbf{c}$ via bond angle variation and reduction of the long $\mathrm{Nb}-\mathrm{Nb}$ contact without necessarily reducing the short metal-metal distance.

A bulk modulus of 380(4) GPa was obtained from an EOS fit to the data of the high-pressure monoclinic phase. This value approaches that of cubic $\mathrm{RuO}_{2}, 399 \mathrm{GPa}$ (Ref. 30 ), and lies in the same range as the superhard materials cubic boron nitride (369 GPa) (Ref. 31) and diamond (442 GPa) ${ }^{32}$ It can be noted that the bulk moduli of the ambient pressure and high pressure phases of metallic and semiconducting $d^{1}, d^{3}$, and $d^{4}$ metal dioxides ${ }^{28,30,33}$ are typically 15 to $30 \%$ greater than the insulating $d^{0}$ and $d^{10}$ cation dioxides ${ }^{18,28}$ for equivalent volumes per ion pair. This is due to the additional metal-metal interactions in the former compounds. The compression of monoclinic $\mathrm{NbO}_{2}$ is also anisotropic. The values of the linear compressibilities obtained on compression, for which there was little variation in the monoclinic angle, are as follows: $\kappa_{a}=6.0(4)$ $\times 10^{-4} \mathrm{GPa}^{-1}, \quad \kappa_{b}=5.0(3) \times 10^{-4} \mathrm{GPa}^{-1}$, and $\kappa_{c}=8.0(4)$ $\times 10^{-4} \mathrm{GPa}^{-1}$.

The volume change at the transition between $\beta-\mathrm{NbO}_{2}$ and monoclinic $\mathrm{NbO}_{2}$ is $10.1(4) \%$ at $8.3 \mathrm{GPa}$ and the difference between the relative volumes at ambient pressure is $11.6(4) \%$. This compares well with the $\Delta V$ between rutile and baddeleyite-type $\mathrm{TiO}_{2}$, which is $9 \%$ at $20.3 \mathrm{GPa}$ (Ref. 14). This large volume change is typical for a transition with an increase in cation coordination number $(\mathrm{CN})$. In the case of the rutile $\rightarrow$ baddeleyite transformation $\mathrm{CN}$ increases from 6 to 7 .

\section{B. Structural relationship between $\mathrm{NbO}_{2}$ phases}

Both $\alpha-\mathrm{NbO}_{2}, \beta-\mathrm{NbO}_{2}$, and high-temperature, rutile-type $\mathrm{NbO}_{2}$ consist of chains of edge-sharing $\mathrm{NbO}_{6}$ octahedra which are directed along $\mathbf{c}$ and cross linked in the $x y$ plane. In the high-temperature, rutile-type phase, the bonded metal ions form straight chains along c, which become zig-zag chains of niobium ions with alternating long and short contacts in $\alpha$ - and $\beta-\mathrm{NbO}_{2}$; see Fig. 4. The niobium ions lie closer to alternate edges of the oxygen octahedra yielding the large dispersion in polyhedral Nb-O distances: $:^{4,11} 1.91-2.25$ $\AA$ for $\alpha-\mathrm{NbO}_{2}$ and 1.904-2.211 $\AA$ for $\beta-\mathrm{NbO}_{2}$.

At the transition from $\alpha-\mathrm{NbO}_{2}$ to $\beta-\mathrm{NbO}_{2}$, cation displacements occur in three out of every four zig-zag chains resulting in a change to the long-range ordering pattern. The differences in long-range order in these two $\mathrm{NbO}_{2}$ polymorphs has been described in detail elsewhere. ${ }^{10}$ In $1 / 4$ of the chains, the cations shift at the transition towards the opposite edge of the octahedra in which they lie. In another $1 / 4$ of the chains, the short and long $\mathrm{Nb}-\mathrm{Nb}$ contacts are inversed. In a further $1 / 4$ of the chains both types of displacement occur, whereas the final 1/4 are unaffected by the phase transition. The principal structural change at the transition thus involves small displacements of $3 / 4$ of the niobium ions, but has very little effect on the overall cell volume. The period length along $\mathbf{a}_{\text {rutile }}$ is 4 in $\alpha-\mathrm{NbO}_{2}$ and 2 in $\beta-\mathrm{NbO}_{2}$. A slight increase in packing efficiency would be expected due to this reduction in period length. $\alpha-\mathrm{NbO}_{2}\left(I 4_{1} / a, Z=32\right)$ and $\beta-\mathrm{NbO}_{2} \quad\left(I 4_{1}, Z=16\right)$ can be related by a common supergroup $^{34} C 4_{2} / m, Z=8$ and the transition can thus be envisaged to occur via a diffusionless process involving small displacements of $3 / 4$ of the cations. The transition is required to be first order, which is confirmed experimentally by the observed coexistence of the two phases at high pressure and the recovery of metastable $\beta-\mathrm{NbO}_{2}$ upon pressure release.

The phase transition from $\beta-\mathrm{NbO}_{2}$ to monoclinic $\mathrm{NbO}_{2}$ is from a distorted rutile-type structure to a distorted baddeleyite-type structure. The following orientational relationships between the rutile-type $(r)\left(P 4_{2} / m n m, Z=2\right)$ and baddeleyite-type $(m) \quad\left(P 2_{1} / c, \quad Z=4\right)$ structures have been proposed: ${ }^{18,35,36}[100]_{m}\left\|[\overline{1} 0 \overline{1}]_{r}, \quad[010]_{m}\right\|[010]_{r}$, $[100]_{m} \|[101]_{r}$. These relationships have permitted a twostep transition pathway ${ }^{18,36}$ passing via the $\mathrm{CaCl}_{2}$-type structure $(P n n m, Z=2)$, which is an orthorhombic distortion of the rutile-type structure, to be envisaged, which involves two group-subgroup descents: $P 4_{2} / m n m, Z=2 \rightarrow P n n m, Z$ $=2 \rightarrow P 2_{1} / c, Z=4$. $\beta-\mathrm{NbO}_{2}$ and monoclinic $\mathrm{NbO}_{2}$ are analogs of rutile and baddeleyite, respectively, in which the unit cells are doubled along all three directions due to the longrange $\mathrm{Nb}-\mathrm{Nb}$ bonding pattern. If the orientational relationships given above hold for these two $\mathrm{NbO}_{2}$ phases, $\beta-\mathrm{NbO}_{2}(\beta)$ could be described using a monoclinic $(m)$ cell $\mathbf{a}_{m}=-\left(\mathbf{a}_{\beta}+\mathbf{c}_{\beta}\right), \quad \mathbf{b}_{m}=\mathbf{b}_{\beta}, \quad$ and $\quad \mathbf{c}_{m}=\mathbf{a}_{\beta}-\mathbf{c}_{\beta} \quad$ with $a$ $=11.3001 \AA, \quad b=9.6076 \AA, \quad c=11.3001 \AA, \quad$ and $\beta$ $=116.47^{\circ}$ at $5.6 \mathrm{GPa}$. The chain direction $\mathbf{c}$ in tetragonal $\beta-\mathrm{NbO}_{2}$ becomes the [101] direction in this monoclinic cell, which is the direction along which the cation chains lie in the high pressure monoclinic phase. The cell constants in monoclinic $\mathrm{NbO}_{2}$ at $8.3 \mathrm{GPa}$ are $a=10.029(2) \AA, \quad b$ $=10.000(2) \AA, c=9.986(2) \AA$, and $\beta=104.29(1)^{\circ}$. The phase transition can be thus envisaged as occurring via a shear with a reduction in monoclinic angle from $116.47^{\circ}$ to $104.29^{\circ}$, a decrease in $a$ and $c$ and a small increase in $b$. This shearing mechanism is consistent with the larger monoclinic angle obtained on compression in the high-pressure phase, 

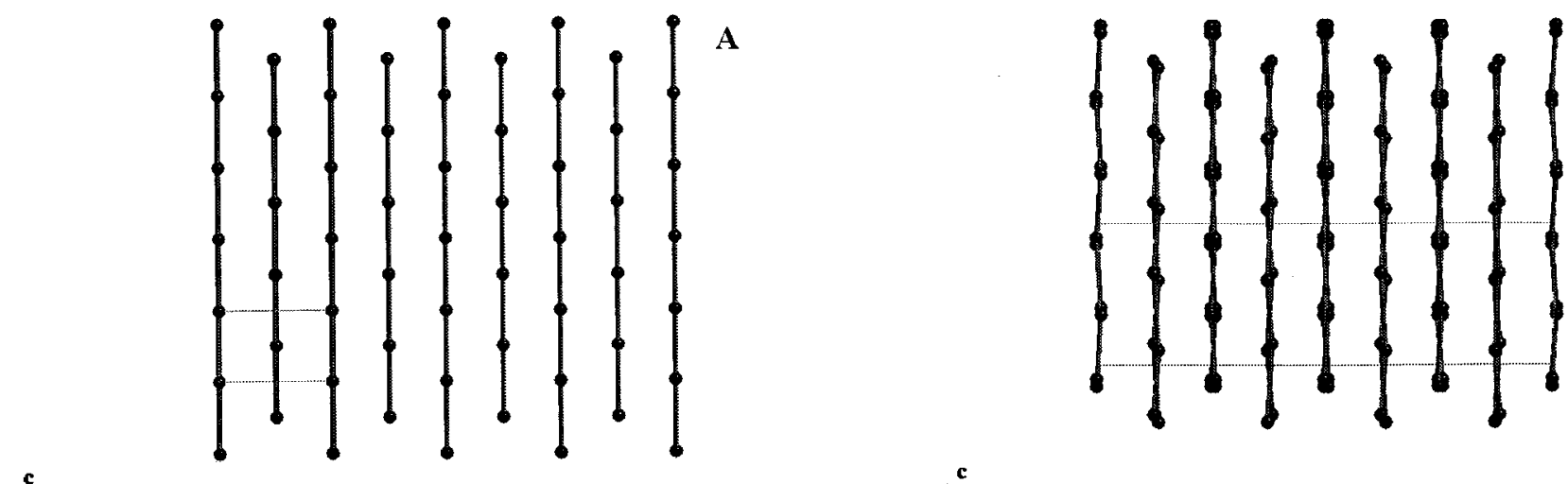

$\mathbf{B}$
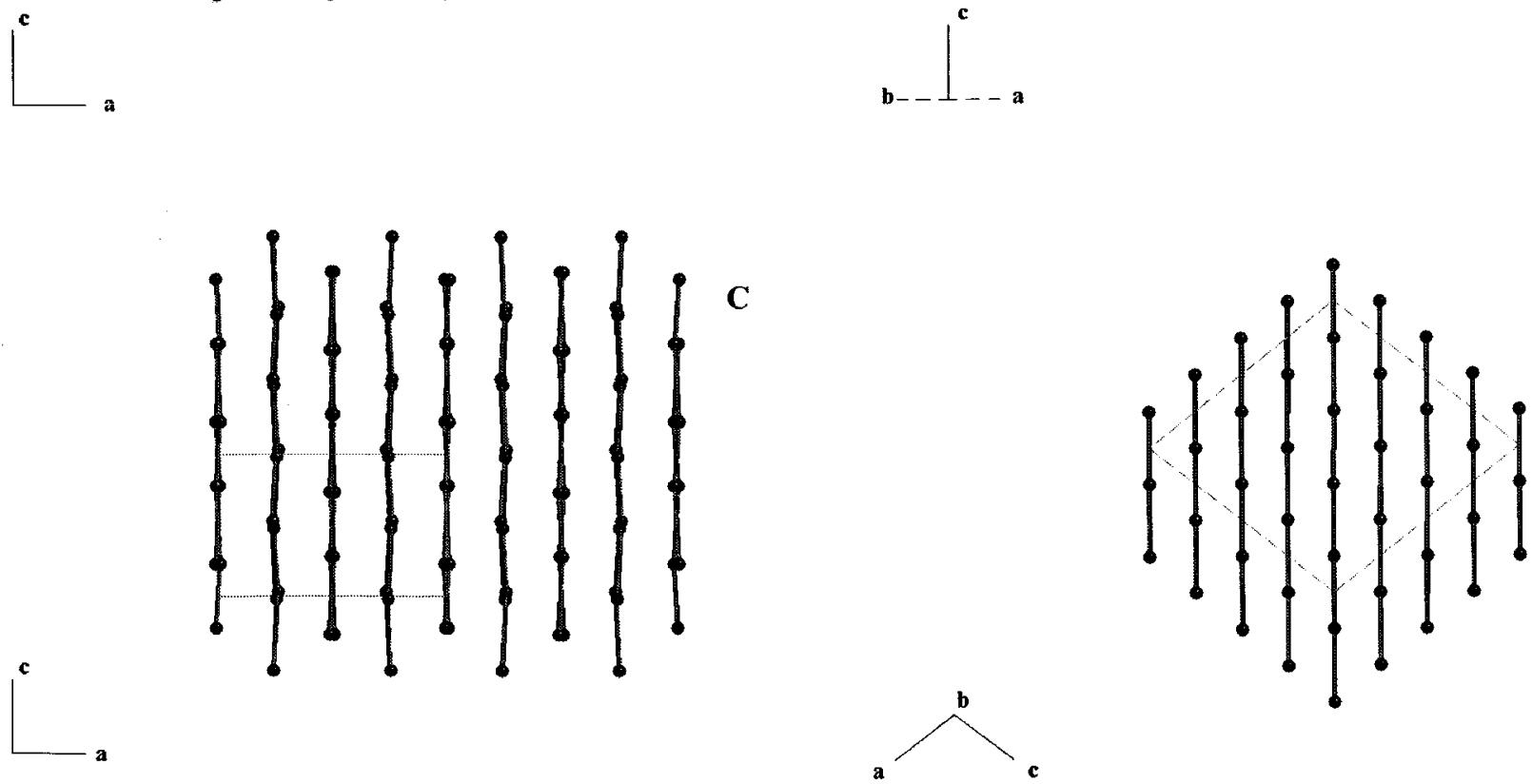

D

FIG. 4. Metal cation substructures of the four polymorphs of niobium dioxide projected as to emphasize their structural similarities: (a) rutile-type $\mathrm{NbO}_{2}$ (010 projection), (b) $\alpha-\mathrm{NbO}_{2}$ (110) projection, (c) $\beta-\mathrm{NbO}_{2}$ (010) projection, and (d) idealized monoclinic $\mathrm{NbO}$ (010 projection). The structure of monoclinic $\mathrm{NbO}_{2}$ is rotated to take into account the orientational relationships described in the text. Directions given for each structure correspond to standard space group settings.

Fig. 2, which could thus be a memory effect connected with defects related to $\beta-\mathrm{NbO}_{2}$. It could therefore be inferred that the measurements obtained upon decompression better represent the true behavior of $\beta$. The positive pressure dependence of $\beta$ impedes the reverse transformation and consequently it is possible to partially quench the high-pressure phase.

As noted previously, the $\beta$ angle in monoclinic $\mathrm{NbO}_{2}$ is larger than in baddeleyite-type $\mathrm{TiO}_{2}$. Tetragonal rutile ${ }^{37}$ $\left(\mathrm{TiO}_{2}\right)$ has the following cell constants at $10.6 \mathrm{GPa} a$ $=4.5096 \AA$ and $c=2.9382 \AA$. Applying the above relationships, the following monoclinic cell can be used to describe rutile-type $\mathrm{TiO}_{2}$ at $10.6 \mathrm{GPa}, a=5.3823 \AA, b=4.5096 \AA$, $c$ $=5.3823 \AA$, and $\beta=113.82^{\circ}$. The observed cell constants for baddeleyite-type $\mathrm{TiO}_{2}$ at $20.3 \mathrm{GPa}$ are $a=4.64 \AA, b$ $=4.76 \AA, c=4.81 \AA$, and $\beta=99.2^{\circ}$. It can be noted that the $\beta$ angle is systematically lower for $\mathrm{TiO}_{2}$, both in the above monoclinic cell used to describe rutile and in the baddeleyite-type phase. The $c / a$ ratio in tetragonal rutiletype structures determines the $\beta$ angle in the corresponding monoclinic cell. At $10.6 \mathrm{GPa}$ in rutile, $c / a=0.6515$, whereas in $\beta-\mathrm{NbO}_{2}$ at $5.6 \mathrm{GPa}$, the $c / a$ ratio of 0.6190 is unusually low due to the short $c$ cell constant resulting from the pres- ence of metal-metal bonds. Consequently, the metal-metal bonds distort the monoclinic cell by increasing $\beta$. This is the case both in the low-pressure and high-pressure phases of $\mathrm{NbO}_{2}$. The proposed transition mechanism thus conserves the cation chain direction and is consistent with the large $\beta$ angle in monoclinic $\mathrm{NbO}_{2}$ relative to baddeleyite-type $\mathrm{TiO}_{2}$.

The structural changes at the two phase transitions in $\mathrm{NbO}_{2}$ are consistent with the resistivity measurements of Okamoto and Minomura. ${ }^{13}$ The $\alpha-\mathrm{NbO}_{2}$ to $\beta-\mathrm{NbO}_{2}$ transition at $5 \mathrm{GPa}$ has little effect on the resistivity as expected as there is essentially no change in the $\mathrm{Nb}-\mathrm{Nb}$ distances. In contrast, at the $\beta-\mathrm{NbO}_{2}$ to monoclinic $\mathrm{NbO}_{2}$ transition at 8 $\mathrm{GPa}$, there is an important change in $\mathrm{Nb}-\mathrm{Nb}$ distances, which begin to approach those in the high-temperature metallic phase and correspondingly, the resistivity was observed to decrease by three orders of magnitude at $8.5 \mathrm{GPa}$. At least one of the short $\mathrm{Nb}-\mathrm{Nb}$ distances in the monoclinic phase is required to be longer than the distances in the metallic hightemperature phase (see Sec. III B), which is consistent with the observation that this phase is a narrow gap semiconductor.

In the $d^{1}$ cation dioxide, $\mathrm{NbO}_{2}$, the additional $d$ electron is involved in metal-metal bonds in both the low-pressure 
and high-pressure phases and the compound follows the phase transition pathway characteristic of $d^{0}$ cation dioxides, ${ }^{14,18,19}$ adopting a baddeleyite-related structure rather than a $\mathrm{Pa} \overline{3}$ structure at high pressure. The effect of the metal-metal bonds is to distort the baddeleyite structure by increasing the $\beta$ angle. A similar increase in $\beta$ is observed in the hydrogen-bonded, baddeleyite-type phase of $\mathrm{Ca}(\mathrm{OD})_{2}$ (Ref. 23).

\section{SUMMARY}

$\alpha-\mathrm{NbO}_{2}$ was found to transform to $\beta-\mathrm{NbO}_{2}$ above $5 \mathrm{GPa}$. $\beta-\mathrm{NbO}_{2}$, which had been previously synthesized by chemical transport reactions, is shown to be a high pressure phase of $\mathrm{NbO}_{2}$. A further transition to a baddeleyite-related phase was observed above $8 \mathrm{GPa}$. Metal-metal bonds are present in all three phases and are responsible for the observed super- structures and the large $\beta$ angle in the monoclinic phase. A transformation mechanism between $\beta-\mathrm{NbO}_{2}$ and the monoclinic phase is proposed which conserves the cation chain direction and is consistent with the large $\beta$ angle relative to baddeleyite-type $\mathrm{TiO}_{2} . \mathrm{NbO}_{2}$ follows the phase transition pathway passing via the baddeleyite structure which is characteristic of $d^{0}$ cation compounds. The relatively large bulk moduli of both $\alpha-\mathrm{NbO}_{2}(230 \mathrm{GPa})$ and the high-pressure monoclinic phase ( $380 \mathrm{GPa}$ ) can be attributed to metal-metal interactions.

\section{ACKNOWLEDGMENT}

We would like to thank the beamline staff of ID30 at the ESRF for making the synchrotron radiation experiment possible in the early commissioning period and for their assistance.
* On leave from Escola de Engenharia and Instituto de Fisica, UFRGS, 91501-970 Porto Alegre, RS, Brazil.

${ }^{1}$ B.-O. Marinder, Acta Chem. Scand. 15, 707 (1961).

${ }^{2}$ R. Pynn, J. D. Axe, and R. Thomas, Phys. Rev. B 13, 2965 (1976).

${ }^{3}$ A. K. Cheetham and C. N. R. Rao, Acta Crystallogr., Sect. B: Struct. Crystallogr. Cryst. Chem. 32, 1579 (1976).

${ }^{4}$ A. A. Bolzan, C. Fong, B. J. Kennedy, and C. J. Howard, J. Solid State Chem. 113, 9 (1994).

${ }^{5}$ R. F. Janninck and D. H. Whitmore, J. Phys. Chem. Solids 27, 1183 (1966).

${ }^{6}$ T. Sakata, K. Sakata, and I. Nishida, Phys. Status Solidi 20, K155 (1967).

${ }^{7}$ K. Sakata, J. Phys. Soc. Jpn. 26, 582 (1969).

${ }^{8}$ S. M. Shapiro, J. D. Axe, G. Shirane, and P. M. Raccah, Solid State Commun. 15, 377 (1974).

${ }^{9}$ G. Bélanger, J. Destry, G. Perluzzo, and P. M. Raccah, Can. J. Phys. 52, 2272 (1974).

${ }^{10}$ H.-J. Schweizer and R. Gruehn, Z. Naturforsch. B 37, 1361 (1982).

${ }^{11}$ D. S. Rimai and R. J. Sladek, Phys. Rev. B 18, 2807 (1978).

${ }^{12}$ S. N. Vaidya, D. K. Joshi, and M. S. Gill, Indian J. Pure Appl. Phys. 17, 802 (1979).

${ }^{13}$ T. Okamoto and S. Minomura, J. Phys. Soc. Jpn. 50, 3661 (1981).

${ }^{14}$ H. Sato, S. Endo, M. Sugiyama, T. Kikegawa, O. Shimomura, and K. Kusaba, Science 251, 786 (1991).

${ }^{15}$ J. Haines, J. M. Léger, and O. Schulte, Science 271, 629 (1996).

${ }^{16}$ J. Haines, J. M. Léger, O. Schulte, and A. Atouf, in High Pressure Science and Technology. Proceedings of the Joint XV AIRAPT and XXXIII EHPRG International Conference, edited by W. A. Trzeciakowski (World Scientific, Singapore, 1996), p. 405.

${ }^{17}$ J. Haines, J. M. Léger, and O. Schulte, J. Phys.: Condens. Matter 8, 1631 (1996).

${ }^{18}$ J. Haines and J. M. Léger, Phys. Rev. B 55, 11144 (1997).

${ }^{19}$ J. M. Léger and J. Haines, Eur. J. Solid State Inorg. Chem. 34, 785 (1997).
${ }^{20}$ H. K. Mao, P. M. Bell, J. W. Shaner, and D. J. Steinberg, J. Appl. Phys. 49, 3276 (1978).

${ }^{21}$ Rodríguez-Carvajal (unpublished).

${ }^{22}$ J. M. Léger, J. Haines, and A. S. Pereira, in Advances in High Pressure Research in Condensed Matter, edited by S. K. Sikka, S. C. Gupta, and B. K. Godwal (NISCOM, New Delhi, 1997), p. 74.

${ }^{23}$ K. Leinenweber, D. E. Partin, U. Schuelke, M. O'Keeffe, and R. B. VonDreele, J. Solid State Chem. 132, 267 (1997).

${ }^{24}$ D. K. Smith and H. W. Newkirk, Acta Crystallogr. 18, 983 (1965).

${ }^{25}$ S. Endo, I. Takenaka, and H. Arashi, in High Pressure Science and Technology, edited by S. L. Schmidt, J. W. Shaner, G. A. Samara, and M. Ross (AIP Press, New York, 1994), p. 371.

${ }^{26}$ F. Birch, J. Geophys. Res. 57, 227 (1952).

${ }^{27}$ W. F. Boyle, J. G. Bennett, S. H. Shin, and R. J. Sladek, Phys. Rev. B 14, 526 (1976).

${ }^{28}$ R. M. Hazen and L. W. Finger, J. Phys. Chem. Solids 42, 143 (1981).

${ }^{29}$ N. L. Ross, J.-F. Shu, R. M. Hazen, and T. Gasparik, Am. Mineral. 75, 739 (1990).

${ }^{30}$ J. Haines and J. M. Léger, Phys. Rev. B 48, 13344 (1993).

${ }^{31}$ E. Knittle, R. M. Wentzcovitch, R. Jeanloz, and M. L. Cohen, Nature (London) 337, 349 (1989).

${ }^{32}$ H. J. McSkimin and P. Andreatch, Jr., J. Appl. Phys. 43, 3944 (1972).

${ }^{33}$ J. Haines, J. M. Léger, and S. Hoyau, J. Phys. Chem. Solids 56, 965 (1995).

${ }^{34}$ W. H. Baur, Z. Kristallogr. 209, 143 (1994).

${ }^{35}$ B. G. Hyde, L. A. Bursill, M. O'Keeffe, and S. Andersson, Nature (London), Phys. Sci. 237, 35 (1972).

${ }^{36}$ A. G. Christy, Acta Crystallogr., Sect. B: Struct. Sci. B49, 987 (1993).

${ }^{37}$ L. C. Ming and M. H. Manghnani, J. Geophys. Res. 84, 4777 (1979). 